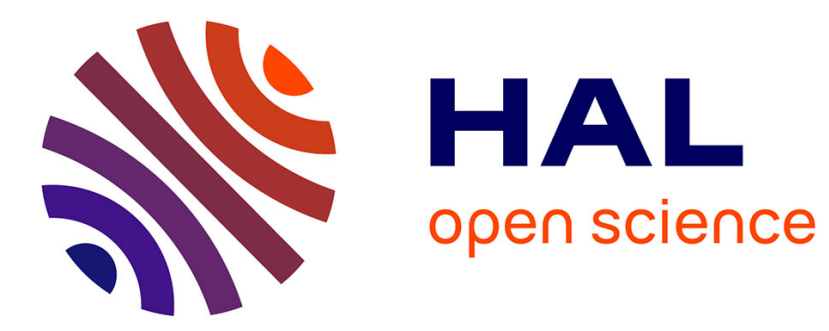

\title{
Improving the Dynamic Accuracy of Elastic Industrial Robot Joint by Algebraic Identification Approach
}

\author{
Marouene Oueslati, Richard Béarée, Olivier Gibaru, George Moraru
}

\section{To cite this version:}

Marouene Oueslati, Richard Béarée, Olivier Gibaru, George Moraru. Improving the Dynamic Accuracy of Elastic Industrial Robot Joint by Algebraic Identification Approach. 1st International Conference on Systems and Computer Science, Aug 2012, LILLE, France. hal-00714545

\section{HAL Id: hal-00714545 \\ https://inria.hal.science/hal-00714545}

Submitted on 4 Jul 2012

HAL is a multi-disciplinary open access archive for the deposit and dissemination of scientific research documents, whether they are published or not. The documents may come from teaching and research institutions in France or abroad, or from public or private research centers.
L'archive ouverte pluridisciplinaire HAL, est destinée au dépôt et à la diffusion de documents scientifiques de niveau recherche, publiés ou non, émanant des établissements d'enseignement et de recherche français ou étrangers, des laboratoires publics ou privés. 


\title{
Improving the Dynamic Accuracy of Elastic Industrial Robot Joint by Algebraic Identification Approach
}

\author{
Marouene Oueslati $^{\dagger, \dagger}$, Richard Béarée ${ }^{\dagger}$, Olivier Gibaru ${ }^{\dagger \dagger}$ and George Moraru ${ }^{\dagger}$ \\ $\dagger$ Laboratory of Science of Information and Systems LSIS-INSM \\ Arts et Métiers ParisTech - 8, Bd Louis XIV, 59046 Lille cedex, France \\ $\ddagger$ Non-A project-team INRIA Lille-Nord-Europe \\ 40, avenue Halley. Bât.A 59650 Villeneuved'Ascq, France \\ Email: marouene.oueslati@inria.fr, \{richard.bearee, olivier.gibaru,george.moraru\}@ensam.eu
}

\begin{abstract}
In this paper, an improvement of the dynamic accuracy of a flexible robot joint is addressed. Based on the observation of the measured axis deformation, a simplified elastic joint model is deduced. In the first step, the non-linear model component's is analyzed and identified in the cases of the gravity bias and the friction term. In the second step, a non-asymptotically algebraic fast identification of the oscillatory behavior of the robot axis is introduced. Finally, the performances of the identification approach are exploited in order to improve the dynamic accuracy of a flexible robot axis. This is done experimentally by the combination of the adaptation of the jerk time profile to reduce the end-point vibration and the model-based precompensation of the end-point tracking error.
\end{abstract}

\section{INTRODUCTION}

Anthropomorphous robots are widely used in many fields of industry to carry out repetitive tasks such as pick and place, welding, assembling, and so on. Due to their flexibility and ability to perform complex tasks in a large workspace, robots are finding their way to realize continuous operations and especially machining applications like, prototyping, cleaning and pre-machining of cast parts ( [4], [16]). Also, a high level precision end-effector trajectory is required in some continuous process. However, the static and dynamic precision are mainly limited by the robot serial structure and the joint stiffness which can induce vibrations of the end effector [13].

In order to control robot motions, we need accurate dynamical models including vibratory phenomena and most of dominating behaviors. These models are function of the geometric parameters, inertial terms and drive chain parameters such as stiffness, viscosity [18], friction and kinematic error issued from the gearbox, ( [8], [19]).

In this paper we present the identification of a robot axis model tacking into account of dominating phenomena [20]. Both of presliding and sliding frictions by means of the Stribeck model are addressed, ( [1], [14]). Moreover, the oscillatory behavior is identified based on algebraic technique introduced by Fliess and Sira-Ramirez ( [5], [6]). The estimation procedure, given by exact formulas in the time domain, leads to a non asymptotic convergence. The identification algorithm is deterministic where the algebraic expressions are written as a function of integrals of system inputs and outputs [11]. In a previous study [15], by comparing this method to classical recursive approach ( [9], [10]), we show its robustness and admits low complexity parameters computation efficiency when measurements are corrupted by noises.

The outline of this paper is as follows. Section 2 describes the robot system in which an elastic joint robot model is addressed in relation with a physical observation of the axis deformation. In section 3, a non linear terms derived from the gravity impact and the joint friction are identified. Here, a new reduced order model is proposed. An on-line estimation of vibratory frequency induced by the joint flexibility and viscosity is developed in section 4 .
Section 5 is devoted the robot axis pre-compensation scheme where experimental results are presented, while Section 6 concludes the paper.

\section{ROBOTIC SYSTEM DESCRIPTION}

The robotic manipulator Stäubli RX-170B is a 6 degree of freedom (DOF) industrial robot with revolute joints. The Stäubli CS8 controller provides the actual joint positions and velocities internally measured through encoders on the motor shafts. The actuator torque is measured through the motor current. The control signals and the state variables are accessible within a sampling time of $4 \mathrm{~ms}$. In order to measure the real position of robot axis, a high-precision API T3 Laser Tracker is used where a Spherically Mounted Retroreflector (SMR) is mounted on the link and guaranteeing an online path tracking. The system resolution is $0.1 \mu \mathrm{m} / \mathrm{m}$ and the angular resolution is about \pm 0.018 arcsec. A well dynamic measurement is ensured thanks to the maximum Tracker acceleration $(>2 g)$. In the presented paper, subsequent modeling and identification analysis are restricted to the second robot axis which is the subject to largest variation of the load with maximum gravitational torque of almost $2150 \mathrm{Nm}$. The robot arms performs trajectories around the $2^{\text {nd }}$ axis with respect to its workspace, while others joints i.e. 1, 3, 4, 5 and 6 are blocked respectively to $[-90,0,0,0,0] \mathrm{deg}$.
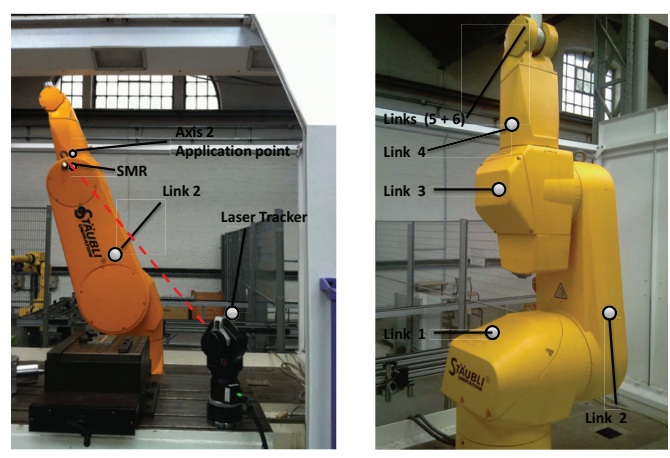

Fig. 1. Stäubli RX-170B robot arm

\section{A. Elastic-joint robot modeling}

1) Dynamic model: The second robot axis can be considered as a two mass coupled system, having the rigid link driven by electrical motor through a rotational joint transmission undergoing a viscoelastic deformation with a constant stiffness $K$ and a viscosity $D$, [17] (see Fig. 2). The flexible joint dynamic is modeled as follows

$$
\begin{gathered}
J \ddot{\theta}_{l}(t)+D \dot{\theta}_{l}(t)+K \theta_{l}(t)=K \theta_{r}(t)-\tau_{r}\left(\theta_{r}(t)\right)-\tau_{f}\left(\dot{\theta}_{r}(t)\right) \\
B \ddot{\theta}_{m}(t)-r^{-1} D \dot{\theta}_{l}(t)-r^{-1} K\left(\theta_{l}-\theta_{r}\right)(t)+r^{-1} \tau_{r}\left(\theta_{r}(t)\right)+r^{-1} \tau_{f}\left(\dot{\theta}_{r}(t)\right)=\tau_{m}
\end{gathered}
$$




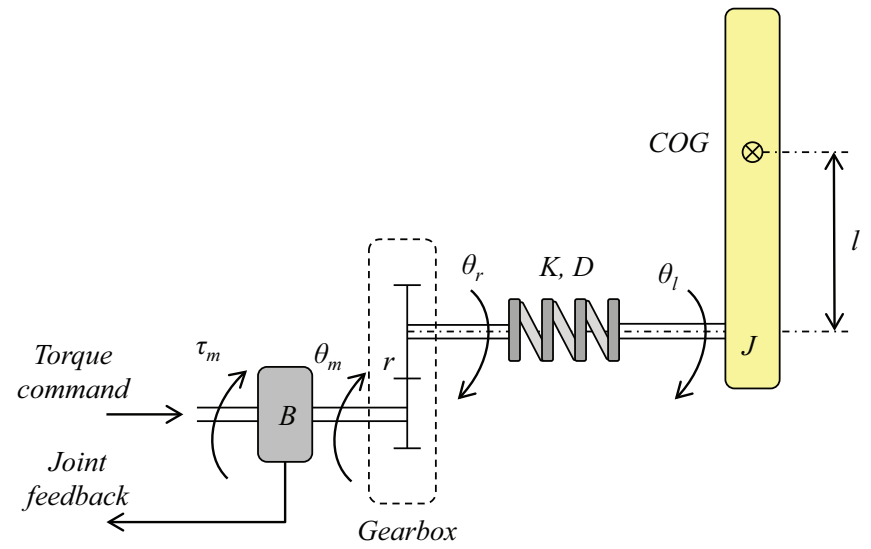

Fig. 2. Elastic robot joint

where $\theta_{l}, \dot{\theta}_{l}$ and $\ddot{\theta}_{l}$ (respectively $\theta_{m}, \dot{\theta}_{m}$ and $\ddot{\theta}_{m}$ ) are the link (respectively the motor) positions, velocities and accelerations. $\theta_{r}$ is the motor position reflected through the second axis gear ratio $r$ with $\theta_{r}=r \theta_{m} . r$ is given by the Stäubli RX-170B datasheet and is equal to 183.03. $J$ denotes the angular mass of the second robot axis under the configuration (see Fig. 1). The actuator inertia is given by $B$. The residual torque defined by (4) is given by $\tau_{r}\left(\theta_{r}\right)$ while $\tau_{f}\left(\dot{\theta}_{r}\right)$ depicts the friction torque defines by the motion of all axes mounted downstream of the second one. Referring to the right-hand side of the equation (2), $\tau_{m}$ denotes the torques supplied by the second axis motor. The motor torque $\tau_{m}$ is determined through the current measurement $i$ :

$$
\tau_{m}(t)=k_{m} i(t), \quad k_{m}=0.51 \quad N . m / r a d
$$

2) Axis deformation: The dynamical model (1),(2) is justified by the following experimental studies. Hence, to capture the joint torsion, the robot axis tracks a position trajectory from -70 deg to 50 deg with successive constant velocities steps as depicted in Fig. 3.

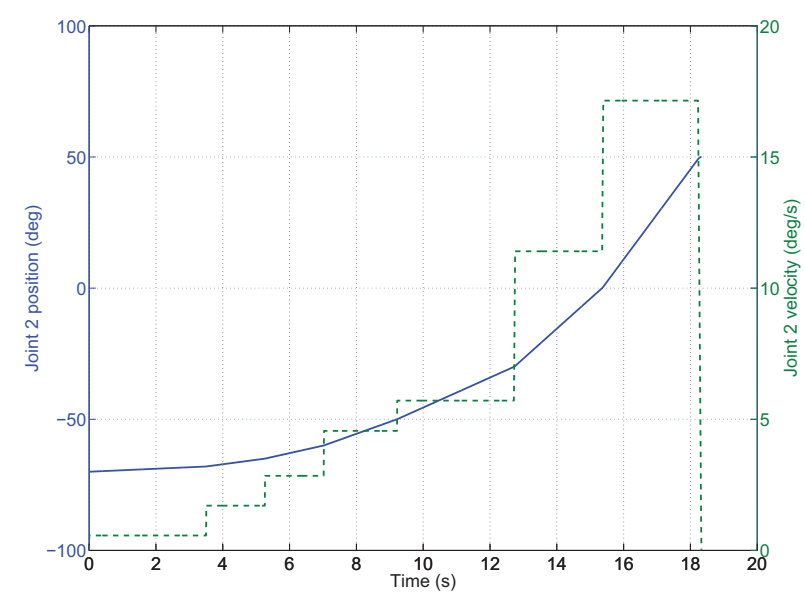

Fig. 3. Joint position reference $\theta_{r}$ and joint velocity reference $\dot{\theta}_{r}$

Fig. 4 depicts the measured tracking errors for $\theta_{r}$ position and the axis deformation $\triangle \theta(t)=\left(\theta_{l}(t)-\theta_{r}(t)\right)$. One notes that the tracking performance of the motor is good despite the presence of acceleration discontinuities. The curves corresponding to the axis deformation show four dominating phenomena which justify the proposed model. By order of contribution, each level can be explained as follows :

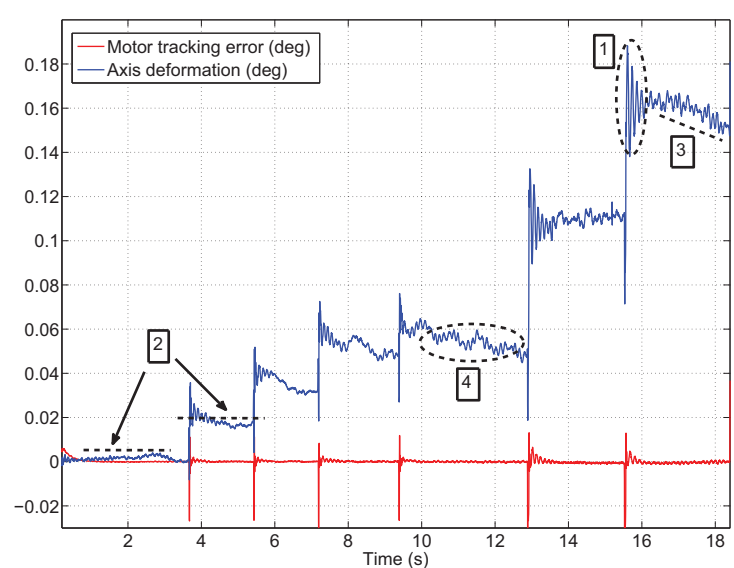

Fig. 4. Measured joint deformation $\triangle \theta$ and joint feedback position error $\theta_{r}$

1) An oscillatory behavior at each velocity changes due to the joint stiffness $K$, the damping $D$ and the moment of inertia $J$. It leads to a low frequency component around $10 \mathrm{~Hz}$.

2) A static error at constant axis velocity dealing with the presence of a viscous friction term in the elastic-joint.

3) A time varying error at constant velocity mainly due to the residual torque.

4) A high frequency component of the axis deformation which correspond to the first harmonic of the gear error.

Conequently, in the next section we are interested in the parameters estimation for the three first phenomena. The gear error model is not developed in this paper.

\section{REDUCED ORDER MODEL FOR ELASTIC-JOINT ROBOT AXIS}

The elastic robot joint behavior results from the superposition of multiple partially coupled phenomena : friction, pre-sliding, gravity and the joint stiffness. Therefore, the experimental setup is designed such that to isolate and decouple these effects for the purpose of parameters estimation.

\section{A. Gravitational-Balancing torques calculation}

The fact that second robot axis is driven by both of actuator and balancing system torques (see Fig. 5), the so-called residual torque emerges from the superposition of previous torques depending on joint position and load mass. This can be expressed as follows:

$$
\tau_{r}=\tau_{g}-\tau_{b}
$$

where, $\tau_{g}$ is the joint gravitational torque, $\tau_{b}$ the torque due to the balancing system and $\tau_{r}$ is the residual torque. The gravitational torque is given by

$$
\tau_{g}\left(\theta_{r}\right)=M_{e q} \times g \times l \times \sin \left(\theta_{r}\right)
$$

where $g$ is the gravitational acceleration. $M_{e q}$ is the sum of masses of each arms making up the robotic system starting from the axis 2 . $l$ matches the radius vector to the gravity center of the total mass from the joint 2 origin as depicted in Fig. 2. The balancing torque $\tau_{b}$ is generated by the action of springs during the rotation of joint 2 (see Fig. 5) and is given by

$$
\tau_{b}\left(\theta_{r}\right)=\left(k_{b} \times x\left(\theta_{r}\right)+P_{c}\right) \times l
$$

in which $k_{b}$ denotes the spring stiffness, $x\left(\theta_{r}\right)$ is the spring displacement in function of the joint position which is nonlinear and $P_{c}$ is the spring preload. These parameters are obtained from robot datasheet's. 

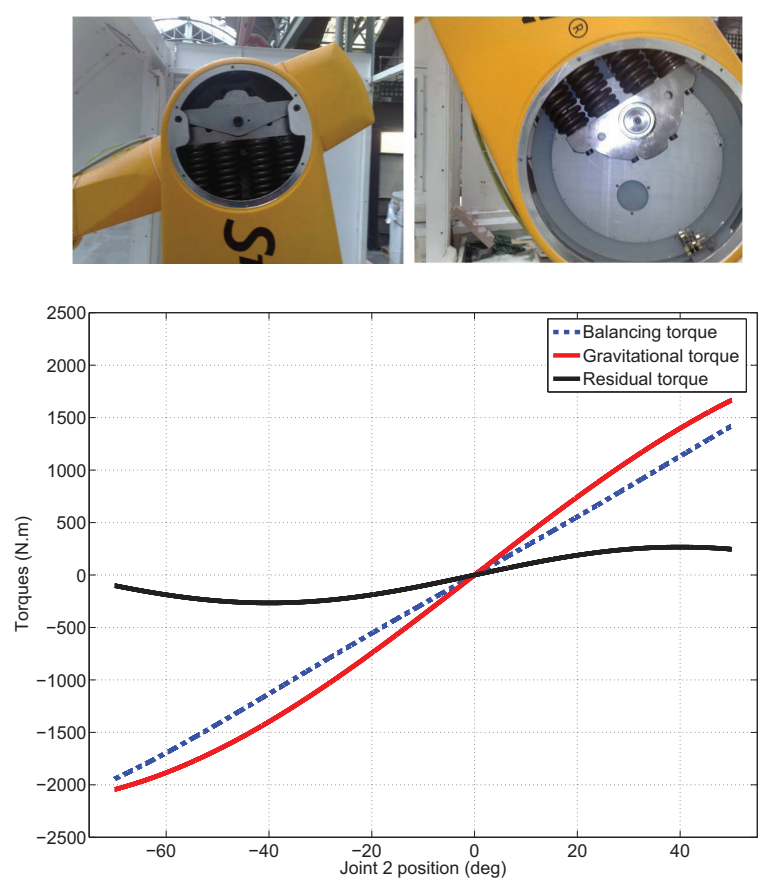

Fig. 5. Balancing system (upper) and Gravitational, Balancing and Residual torques (below)

\section{B. Friction model and Pre-sliding friction estimation}

1) Friction model: Aiming that gravity impact will not affect joint motion, the link is operated in the neighborhood of the residual torque free position $\theta_{r} \approx 0 \mathrm{deg}$ (see Fig. 5). The joint is driven back and fourth at constant velocities $[ \pm 0.004 \pm 0.006, \ldots, \pm 0.2] \mathrm{rad} / \mathrm{s}$ in a bidirectional drive experiment. Friction torque values correspond to residual joint torque subtracted from applied motor torque seen after the joint transmission $r$. The joint friction torque $\tau_{f}\left(\dot{\theta}_{r}\right)$ is fitted by means of the Stribeck model [3] in the sliding regime (Fig. 6). The model for friction is given by

$$
\tau_{f}\left(\dot{\theta}_{r}\right)=\left(f_{c}+\left(f_{s}-f_{c}\right) e^{-\left|\frac{\dot{\theta}_{r}}{\theta_{r_{s}}}\right|^{2}}\right) \operatorname{sgn}\left(\dot{\theta}_{r}\right)+f_{v} \dot{\theta}_{r}
$$

It must be noted that Stribeck characteristic curves i.e negative and

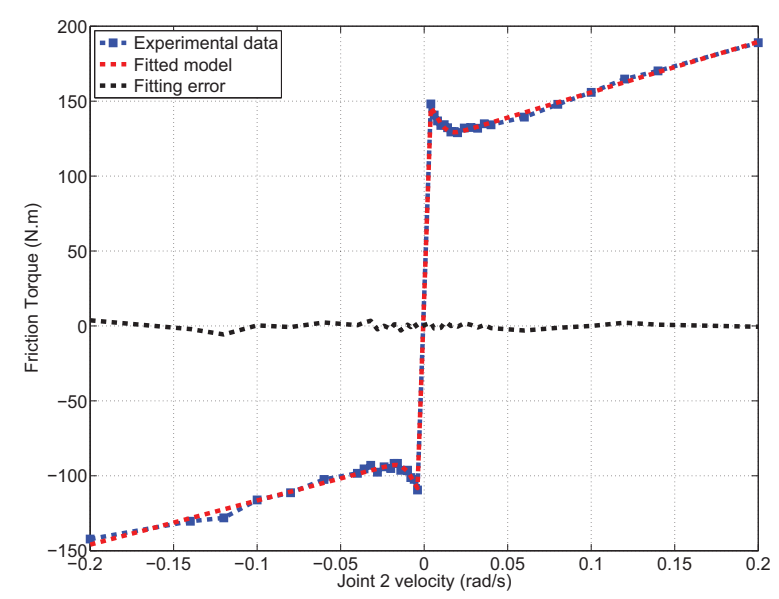

Fig. 6. Experimental friction torque and fitted Stribeck curve

positive velocities, are fitted to the experimental data with a Gauss-
Newton algorithm that minimizes the summed squares error by the instruction lsqcurvefit in the Matlab package. As it is shown in Fig. 6 , friction torque was noticed to be non-symmetric at positive and negative velocities as it is depicted in TABLE I.

2) Pre-sliding friction estimation: The static friction (pre-sliding regime) is identified by means of an experiment with a constant very low velocity of $5.93110^{-5} \mathrm{rad} / \mathrm{s}$. During the process, the velocity reference causes in the contacting surfaces of the joint transmission a cyclic motion in function of the joint displacement at the initialization of the axis movement, as shown in Fig. 7. Hence, the pre-sliding friction involved in the Stribeck model (7), is well identified and verified thanks to the experimental identification and the curve fitting algorithm. It must be noted that according to the estimation algorithm, $f_{S_{\text {Model }}}=148.5443 \mathrm{Nm}$ and by referring to the experimental pre-sliding test, $f_{s_{\text {Exper }}} \approx 150 \mathrm{Nm}$.

TABLE I

STRIBECK FRICTION COEFFICIENTS

\begin{tabular}{|c|c|c|c|}
\hline \multicolumn{5}{|c|}{ Joint 2 positive velocity } \\
\hline$f_{c}=122.19 \mathrm{Nm}$ & $f_{s}=148.54 \mathrm{Nm}$ & $f_{v}=336.73 \mathrm{Nm} . \mathrm{s} / \mathrm{rad}$ & $\dot{\theta}_{r_{s}}=0.009 \mathrm{rad} / \mathrm{s}$ \\
\hline \hline \multicolumn{4}{|c|}{ Joint 2 negative velocity } \\
\hline$f_{c}=87.02 \mathrm{Nm}$ & $f_{s}=111.38 \mathrm{Nm}$ & $f_{v}=294.44 \mathrm{Nm} . \mathrm{s} / \mathrm{rad}$ & $\dot{\theta}_{r_{s}}=0.009 \mathrm{rad} / \mathrm{s}$ \\
\hline
\end{tabular}

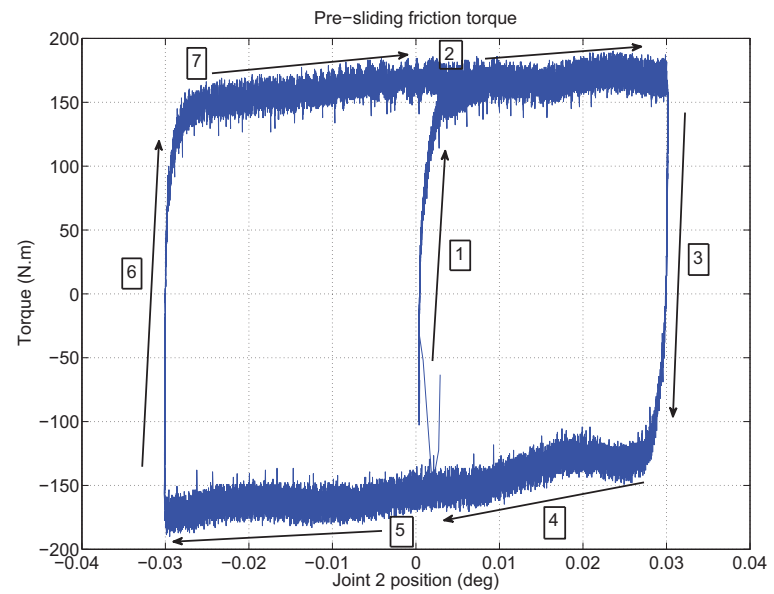

Fig. 7. Pre-sliding friction at $\theta_{r}=0 \mathrm{deg}$

\section{Angular mass, Stiffness and Damping estimation}

In this subsection we are interested in the estimation of the moment of inertia $J$, the axis stiffness $K$ and the damping coefficient $D$. The identification procedure are performed with respect to the previous robot configuration where joints $1,3,4,5$ and 6 are fixed respectively to $[-90,0,0,0,0] \mathrm{deg}$.

1) Angular mass identification: The estimation procedure consists in performing a constant acceleration profile where the robot axis tracks a first order velocity polynomial's. Assuming that rotor inertia $B$ in (2) is obtained from the manufacturer data, $J$ is then deduced by means of the division of acting torques in the joint side through the acceleration vector. In Fig. 8, experimental result of $J$ identification is revealed where the inertial torque is computed from the sum of captured joint friction (6), gravitational impact (4) subtracted from the acting motor torque.

2) Stiffness and damping estimation: Focus on joint torsion (Fig. 9), a second order model can be locally identified where the contributed parameters are limited to $J, K$ and $D$. An estimation of the stiffness and the damping coefficients is performed based on the 


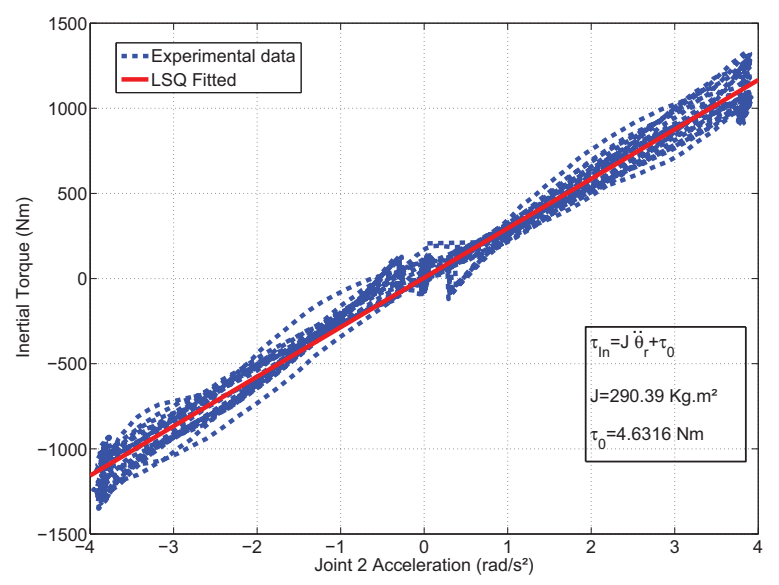

Fig. 8. Joint 2 moment of inertia : experimental data and fitted model

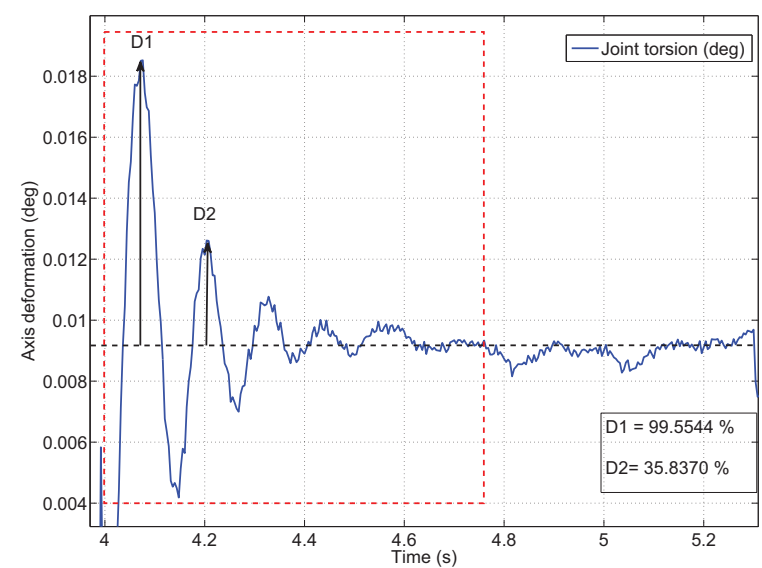

Fig. 9. Robot axis deformation

computation of the logarithmic decrement $\delta$, damping ratio $\zeta$ and the vibration period $T$.

$$
\left\{\begin{array} { c } 
{ \zeta = 0 . 1 6 2 6 } \\
{ \omega _ { 0 } = 6 0 . 9 6 8 \mathrm { rad } / \mathrm { s } }
\end{array} \Rightarrow \left\{\begin{array}{l}
K=J \omega_{0}^{2}=1.079410^{6} \mathrm{~N} . \mathrm{m} / \mathrm{rad} \\
D=2 \zeta \sqrt{K J}=5.7575 \quad 10^{3} \mathrm{~N} . \mathrm{m} . \mathrm{s} / \mathrm{rad}
\end{array}\right.\right.
$$

\section{Reduce order model}

One notes that the motor tracking error does not exceed $0.5 \%$ of the reference position signal (Fig. 4). Indeed, we can conclude that the controller tracking error can be neglected as compared to the end-point tracking error i.e the joint deformation $\triangle \theta$. This realistic hypothesis conducts to a reduce order model which can be limited to (1). The input data is then the motor position reflected through the nominal value of the gear ratio $r$ i.e $r \theta_{m}$ measured with the robot controller and the output is the link position $\theta_{l}$ measured with the laser tracker device. Fig. 10 depicts the model validation scheme.

\section{NON-ASYMPTOTIC METHOD FOR REAL-TIME ESTIMATION OF VIBRATION FREQUENCY}

In this section, we provide the reader with the estimation of the parameters involved in the second order model of the robot axis, i.e. the damping ratio $\zeta$ and the resonance frequency $\omega_{0}$. The fundamental developments are based on the module theoretic approach to linear systems proposed by ( [5], [6], [11]). This technique was

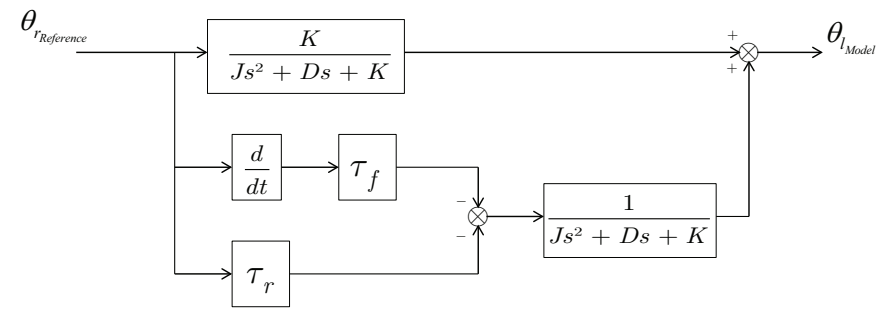

Fig. 10. Validation block diagram

compared to classical recursive approach as shown in [15]. It allows us to validate the physical identification of the involved coefficients in the previous subsection. The non-asymptotic identification of these parameters allows the user to capture the axis' dynamics for each time step on the one hand and to design a feed-forward control to compensate the end-point tracking error on the other hand. Moreover, the real-time ability of the estimation algorithm is promising for counteract the time variations of parameters in robotic applications.

\section{A. Method description}

Since the main contribution of the axis's dynamics is issued from its oscillatory behavior (damping oscillations Fig. 9), it will be shown that the two parameters $\zeta$ and $\omega_{0}$ can be estimated under the transient response of the joint deformation using equation (9).

$$
J \ddot{\theta}_{l}(t)+D \dot{\theta}_{l}(t)+K \theta_{l}(t)=K \theta_{r}(t)
$$

Assuming that $\zeta$ is the damping ratio and $\omega_{0}$ is the resonance frequency, (9) can be rewritten as

$$
\ddot{\theta}_{l}(t)=-\lambda_{1} \dot{\theta}_{l}(t)-\lambda_{2} \theta_{l}(t)+\lambda_{2} \theta_{r}(t)
$$

where $\lambda_{1}=2 \zeta \omega_{0}$ and $\lambda_{2}=\omega_{0}^{2}$. Indeed, using an algebraic approach for (10), it is possible to express the parameters $\lambda_{1}$ and $\lambda_{2}$ as a function of the measured input $\theta_{r}$ and the output $\theta_{l}$.

Assuming that $\hat{\theta}_{l}(s)$ and $\hat{\theta}_{r}(s)$ are the Laplace transform of $\theta_{l}(t)$ and $\theta_{l}(t)$, we consider the Laplace transform of (10)

$$
s^{2} \hat{\theta}_{l}(s)-s \theta_{l}(0)-\dot{\theta}_{l}(0)=-\lambda_{1}\left(s \hat{\theta}_{l}(s)-\theta_{l}(0)\right)-\lambda_{2}\left(\hat{\theta}_{l}(s)-\hat{\theta}_{r}(s)\right)
$$

In order to eliminate the initial condition, we apply the derivative operator with respect to $s$ two times. It leads to

$$
\begin{aligned}
s^{2} \frac{d^{2} \hat{\theta}_{l}(s)}{d s^{2}}+4 s \frac{d \hat{\theta}_{l}(s)}{d s}+2 \hat{\theta}_{l}(s) & =\lambda_{1}\left(-s \frac{d^{2} \hat{\theta}_{l}(s)}{d s^{2}}-2 \frac{d \hat{\theta}_{l}(s)}{d s}\right) \\
& +\lambda_{2}\left(\frac{d^{2} \hat{\theta}_{l}(s)}{d s^{2}}-\frac{d^{2} \hat{\theta}_{l}(s)}{d s^{2}}\right)
\end{aligned}
$$

It remind that differentiation with respect to $s$ in the operational domain results in a multiplication by $-t$ in the time domain. The multiplication by $s$ in the operational domain leads to the derivation in the time domain. As it well known that derivation amplify the high frequency and then the noise contribution, so the application of the estimator (12) is not convenient. Thus, we divide (12) by $s^{3}$ in order to cancel the derivation terms and obtain a relationship with integral operators. Consequently, applying the classical cauchy formula, we compute the inverse Laplace transform and return to time domain as follows:

$$
\mathscr{L}^{-1}\left(\frac{1}{s^{m}} \frac{d^{n} \theta(s)}{d s^{n}}\right)=\frac{(-1)^{n}}{(m-1) !} \int_{0}^{t}(t-\tau)^{m-1} t^{n} \theta(\tau) d \tau=P_{m, n}^{\theta}(t)
$$

It yields,

$$
\begin{aligned}
P_{1,2}^{\theta_{l}}(t)+4 P_{2,1}^{\theta_{l}}(t)+2 P_{3,0}^{\theta_{l}}(t) & =\lambda_{1}\left(-P_{2,2}^{\theta_{l}}(t)-2 P_{3,1}^{\theta_{l}}(t)\right) \\
& +\lambda_{2}\left(P_{3,2}^{\theta_{l}}(t)-P_{3,2}^{\theta_{r}}(t)\right)
\end{aligned}
$$


Then, we have to generate one more equation in order to obtain a linear system so as to identify $\lambda_{1}$ and $\lambda_{2}$. It is well known that integration in time domain is a linear operation, thus, by taking (12) and dividing it one time with respect to $s^{4}$, one gets the following linear system in the time domain

$$
\begin{aligned}
P_{2,2}^{\theta_{l}}(t)+4 P_{3,1}^{\theta_{l}}(t)+2 P_{4,0}^{\theta_{l}}(t) & =\lambda_{1}\left(-P_{3,2}^{\theta_{l}}(t)-2 P_{4,1}^{\theta_{l}}(t)\right) \\
& +\lambda_{2}\left(P_{4,2}^{\theta_{l}}(t)-P_{4,2}^{\theta_{r}}(t)\right)
\end{aligned}
$$

Equations (13) and (14) allow to obtain expressions of the estimate of $\lambda_{1}$ and $\lambda_{2}$.

$$
P(t) \Theta=Q(t)
$$

where $\Theta=\left[\begin{array}{ll}\lambda_{1} & \lambda_{2}\end{array}\right]^{T}, P(t)=\left(\begin{array}{cc}-P_{2,2}^{\theta_{l}}(t)-2 P_{3,1}^{\theta_{l}}(t) & P_{3,2}^{\theta_{l}}(t)-P_{3,2}^{\theta_{r}}(t) \\ -P_{3,2}^{\theta_{l}}(t)-2 P_{4,1}^{\theta_{l}}(t) & P_{4,2}^{\theta_{l}}(t)-P_{4,2}^{\theta_{r}}(t)\end{array}\right)$ and, $Q(t)=\left(\begin{array}{l}P_{1,2}^{\theta_{l}}(t)+4 P_{2,1}^{\theta_{l}}(t)+2 P_{3,0}^{\theta_{l}}(t) \\ P_{2,2}^{\theta_{l}}(t)+4 P_{3,1}^{\theta_{l}}(t)+2 P_{4,0}^{\theta_{l}}(t)\end{array}\right)$

For a given value $t>0$ and if $P(t)$ is invertible, then, an estimation of $\Theta$ is obtained by

$$
\left(\begin{array}{l}
\lambda_{1} \\
\lambda_{2}
\end{array}\right)=P(t)^{-1} Q(t)
$$

Note that (13) and (14) are expressed in terms of a linear combination of iterated convolution integrals over process input and output. However, there is two unknown parameters to estimate.

\section{B. Implementation}

Aiming to estimate the unknown parameters, the algebraic estimator i.e the iterated integrals present in (13) and (14) have been discretized using the trapezoidal rule [2]. Besides, in order to estimate $\hat{\lambda}_{1}$ and $\hat{\lambda}_{2}$ not asymptotically, integrating over a short time interval $[0, t]$ provides an online parametric estimator. Meanwhile, $t$ cannot obviously be taken arbitrary small even in a noise free context [15]. A lower bound for $t$ has been formally characterized in [7], within the framework of nonstandard analysis. Let us quote that the estimation time $T_{w}$ may be small, resulting in fast estimation. The experience shows that a sliding window $t=20 T_{S}$ with $T_{S}$ be the sampling time equals to $0.004 \mathrm{~s}$ gives good results. From the experimental
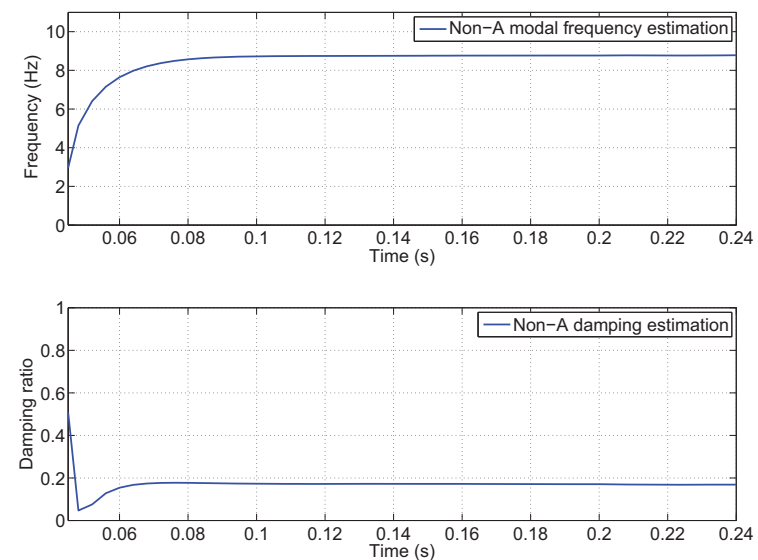

Fig. 11. Experimental estimation of the parameters

data, the experience shows that after 0.08 seconds the parameters have converged. Besides, one gets an estimation of $\hat{f}_{0}=\frac{\hat{\omega}_{0}}{2 \pi}=8.972$ $\mathrm{Hz}$ and $\hat{\zeta}=0.1722$. Indeed, when they are compared to the ones obtained by physical identification method (see section 3.C). We have an estimation error less than $7.35 \%$ for $\omega_{0}$ and about 5.57 $\%$ for $\zeta$.

\section{Model validation}

From the protocol test defined in Fig. 3, we depict in Fig. 12 the comparison between the measured joint deformation and the simulated one through the identified model response. The parametric identification via the non-asymptotic approach for the linear part of the reduce order model $\left(\omega_{0}, \zeta\right)$ and the non-linear contribution derived from the gravity impact and the friction term, converges as well as possible to the real process behavior. Furthermore, the model presents a good fit equal to $82 \%$. The modeling error can be explained through some uncertainties in the estimation of the friction and the calculation of the residual torques where the balancing torque include the pre-load variation. In addition, the performances of the
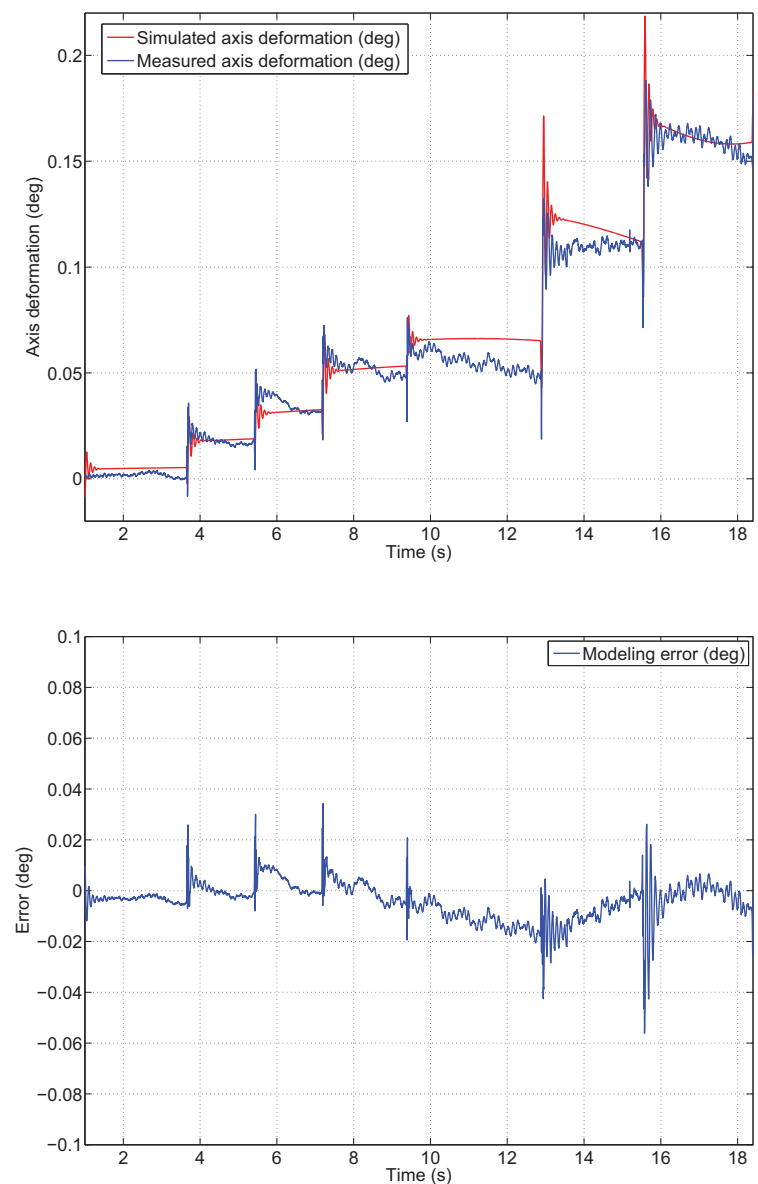

Fig. 12. Axis deformation model validation (upper) and modeling error (below)

estimation's algorithm i.e robustness and convergence rapidity can be strongly exploited so as to improve the dynamic accuracy of the robot axis.

\section{TOWARDS IMPROVING THE DYNAMIC ACCURACY OF THE ROBOT AXIS VIA TRAJECTORY PRE-COMPENSATION}

In order to reduce the second robot axis deformation, the strategy of joint pre-compensation consists in designing a feed-forward control so as to anticipate the end-point tracking error based on the identified model:

$$
\theta_{m_{\text {command }}}(t)=\theta_{m_{\text {reference }}}(t)-\triangle \theta_{\text {simulated }}(t)
$$


where, $\theta_{m_{\text {command }}}$ is the new joint command anticipation, $\theta_{m_{\text {reference }}}$ is the theoretical joint reference and $\triangle \theta_{\text {simulated }}$ the simulated joint deformation obtained from the axis model.

\section{A. Experimental validation}

The validation experiments is performed under the joint 2 position profile (Fig. 13), where the jerk profile (acceleration time derivative) is chosen so as to reduce the oscillatory behavior of the studied axis. This is done settled on the natural frequency estimation given by the identification approach (section 4). For further details about jerk time methodology, see [13]. Fig. 14 depicts the evolution of
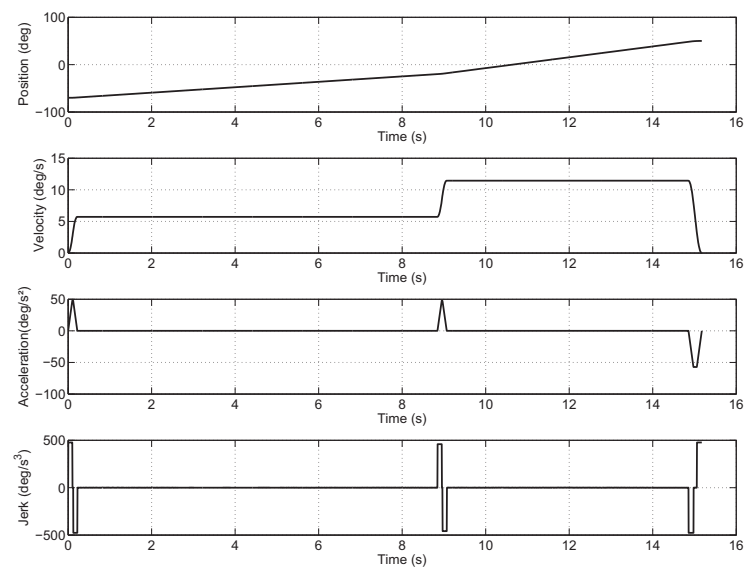

Fig. 13. Joint 2 position profile and its time derivatives resulting from path planning algorithm

the joint deformation before and after trajectory compensation. One can concludes that the amplitude of $\triangle \theta$ is at least two times smaller after trajectory anticipation and leads to a significant reduction of the end-point tracking error. We must takes into consideration that the compensation law do not integrates also all the error sources, such as the kinematic error of the gear [8], [19], which will be investigated and integrated in a future work.

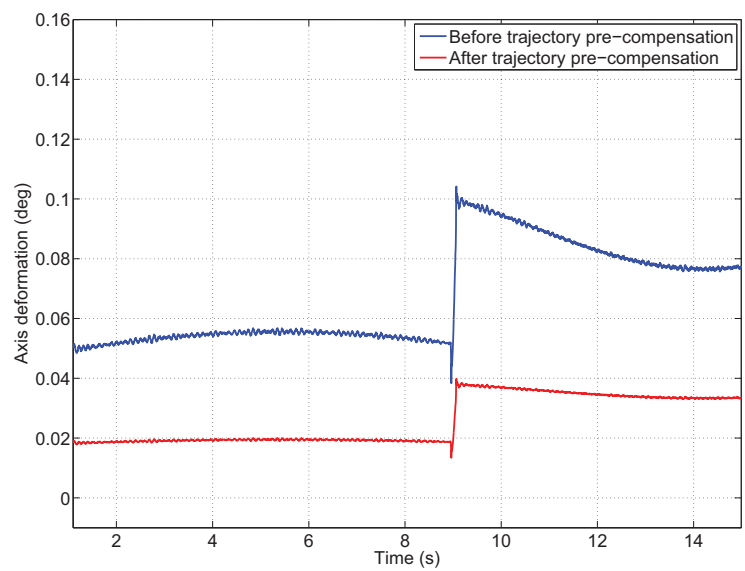

Fig. 14. $\Delta \theta$ without and with pre-compensation of trajectory given in Fig. 13

\section{CONCLUSion}

The objective of this paper was to improve the dynamic accuracy of an elastic industrial robot joint. A reduce global model composed of a linear and a non-linear parts was investigated and developed based on experiments. Also, our approach provides a mean to capture the major phenomena acting on the joint deformation. The non-linear terms derived from the gravity impact and the joint friction in both pre-sliding and sliding regimes of system drive are described. It has been presented a non-asymptotic estimator to the fast and reliable identification of dynamical parameters of the linear part of the model. The estimation algorithm is able to estimate simultaneously and in a fast way (less than $80 \mathrm{~ms}$ ) the modal frequency and the damping ratio. The computational complexity of the estimator is low, robust and the experimental results are promising. Moreover, based on the performances of the identification technique, a pre-compensation scheme of the end-point tracking error was designed. Hence, using the identified model, the experiments show that the axis trajectories are well tracked after joint position anticipation. As improvement, it will be interesting to investigate the spatial varying error due to the kinematic error of the gear and to include it into the model. Thus, the identified model and compensation strategies are useful for the control design that is applicable to all robots of similar architecture.

\section{REFERENCES}

[1] G. Abba and P. Sardain. Friction modelling of a robot transmission chain with identification in mind. Mecaniques \& Industries, 4:391-396, 2003.

[2] M. Abramowitz and I.A. Stegun. Handbook of mathematical functions. Dover 1965.

[3] N.A. Bompos, P.K. Artemiadis, A.S. Oikonomopoulos, and K.J. Kyriakopoulos Modeling, full identification and control of the mitsubishi pa-10 robot arm. 2007 IEEE/ASME international conference on Advanced intelligent mechatronics, pages 1-6, 4-7, Sept 2007.

[4] J.J. Craig. Introduction to Robotics: Mechanics and Control. Addison - Wesley, Reading, 1986.

[5] M. Fliess and H. Sira-Ramirez. An algebraic frameworks for linear identification. Esaim cont. opt. cal. variat., 9, 2003.

[6] M. Fliess and H. Sira-Ramirez. Closed-loop parametric identification for continuous-time linear systems via new algebraic technique. Identification of Continuous-time Models from sampled Data, pages 362-391, 2008.

[7] M. Fliess. Analyse non standard du bruit,. CRAS, Serie 1, Mathmatiques, 342, 797-802, 2006

[8] F.H. Ghorbel, P.S. Gandhi, and F. Alpeter. On the kinematic error in harmonic drive gears. Journal of Mechanical Design, Transactions Of the ASME, 123(1):90-97, 2001.

[9] I.D. Landau. Identification et commande des systemes. 2e dition revue et augmente. Hermes, 1993. 534p.

[10] L. Ljung and V. Söderstmö. Theory and Practice of Recursive Identification. The MIT Press, 1983.

[11] M. Mboup. Parameter estimation via differential algebra and operational calculus. Manuscript, 2004. Available at http://hal.inria.fr/inria-00138294.

[12] M. Mboup, C. Join, and M. Fliess. A revised look at numerical differentiation with an application to nonlinear feedback control. The 15th Mediterran Conference on Control and Automation-MED'2007, 27-29, Jul 2007.

[13] A. Olabi, R. Bearee, O. Gibaru, and M. Damak. Feedrate planning for machining with industrial six-axis robots. Control Engineering Practice, 18(5):471-482, 2010.

[14] H. Olsson, H.J. Astrom, C.C. De Wit, M Gafvert, and P Lischinsky. Friction models and friction compensation. Eur. J. Control, 4, 3:176-195, 1998.

[15] M. Oueslati, S. Thiery, O. Gibaru, R. Bearee, and G. Moraru. On algebraic approach for msd parametric estimation. Integrated Modeling and Analysis in Applied Control and Automation conference, pages 83-91, Sep 2011.

[16] R. Paul. Robot manipulators: Mathematics, programming and control. Technical report, MIT Press, Cambridge, 1982.

[17] B. Sicilinao and O. Khatib. Handbooks of Robotics. ISBN: 978-3-540-23957-4, e-ISBN: 978-3-540-30301-5. Springer, 2008. $1611 \mathrm{p}$.

[18] M.W. Spong. Modeling and control of elastic joint robots. ASME J. of Dynamic Systems, Measurement, and control, 109(4):310-319, 1985.

[19] D.T. Tuttle. Understanding and modeling the behavior of a harmonic drive gear transmission. Technical report, Massachusetts Institute of Technology, 1992.

[20] E. Wernholt and S. Gunnarsson. Nonlinear identification of a physically parameterized robot model. Technical Report LiTH-ISY-R-2739, Department of Electrical Engineering, Linkping University, SE-581 83 Linkping, Sweden, Aug 2006. 